\title{
Elimination of Second-Harmonics in CMUTs using Square Pulse Excitation
}

Lei, Anders; Diederichsen, Søren Elmin; Hansen, Sebastian Molbech; Stuart, Matthias Bo; Bagge, Jan Peter; Jensen, Jørgen Arendt; Thomsen, Erik Vilain

\section{Published in:}

Proceedings of 2016 IEEE International Ultrasonics Symposium

Link to article, DOI:

10.1109/ULTSYM.2016.7728824

Publication date:

2016

Document Version

Peer reviewed version

Link back to DTU Orbit

Citation (APA):

Lei, A., Diederichsen, S. E., Hansen, S. M., Stuart, M. B., Bagge, J. P., Jensen, J. A., \& Thomsen, E. V. (2016). Elimination of Second-Harmonics in CMUT's using Square Pulse Excitation. In Proceedings of 2016 IEEE International Ultrasonics Symposium IEEE. https://doi.org/10.1109/ULTSYM.2016.7728824

\section{General rights}

Copyright and moral rights for the publications made accessible in the public portal are retained by the authors and/or other copyright owners and it is a condition of accessing publications that users recognise and abide by the legal requirements associated with these rights.

- Users may download and print one copy of any publication from the public portal for the purpose of private study or research.

- You may not further distribute the material or use it for any profit-making activity or commercial gain

- You may freely distribute the URL identifying the publication in the public portal 


\title{
Elimination of Second-Harmonics in CMUTs using Square Pulse Excitation
}

\author{
Anders Lei*, Søren Elmin Diederichsen*, Sebastian Molbech Hansen*, Matthias Bo Stuart ${ }^{\dagger}$, \\ Jan Peter Bagge ${ }^{\ddagger}$, Jørgen Arendt Jensen ${ }^{\dagger}$, and Erik Vilain Thomsen* \\ * Department of Micro and Nanotechnology, Technical University of Denmark, DK-2800 Kgs. Lyngby, Denmark \\ †Center for Fast Ultrasound Imaging, Department of Electrical Engineering, Technical University of Denmark, \\ DK-2800 Kgs. Lyngby, Denmark \\ ${ }_{\ddagger}^{\ddagger}$ BK Ultrasound, Herlev, Denmark
}

\begin{abstract}
The harmonic imaging mode is today a fundamental part of ultrasound imaging; it is not only used for suppressing the grating lobe artifact, but also to reduce many other acoustical artifacts in the ultrasound image. A vital performance parameter for accepting CMUT probes as a clinical usable transducer technology is, that it can support harmonic imaging. The large bandwidth of the CMUT is a clear advantage for harmonic imaging, but the inherent nonlinear behavior of the CMUT poses an issue as it is difficult to dissociate the harmonics generated in the tissue from the harmonic content of the transmitted signal. This work presents how proper pulse coding of a bipolar pulser, which is present in most commercial ultrasound scanners, can reduce the intrinsic generated harmonic to fundamental pressure amplitude ratio to below $-\mathbf{3 5} \mathrm{dB}$, making CMUT probes usable for clinical applications.
\end{abstract}

\section{INTRODUCTION}

Capacitive micromachined ultrasonic transducers (CMUTs) are a promising alternative to piezoelectric transducers for medical ultrasound. Among the advantages are design flexability, compatibility with CMOS, and ease of fabrication resulting in a possible lowering of transducer cost. The main selling point is, however, the low mechanical impedance of the CMUT, which results in a large immersion bandwidth and therefore high axial resolution. A consequence of the low mechanical impedance is a lower output pressure compared to PZT, which for conventional imaging is counterbalanced by a higher receive sensitivity. The large bandwidth also makes CMUTs interesting for nonlinear imaging, where pressure is transmitted at the fundamental frequency and higher order harmonics are received. The CMUTs, however, have some characteristics that cause issues in the usability for efficient nonlinear imaging. The transmit pressure of a simplified 1-D CMUT is proportional to the electrostatic force [1]:

$$
F_{\mathrm{e}}=\frac{1}{2} \epsilon_{0} A \frac{V^{2}(t)}{\left(g_{\mathrm{eff}}-w(t)\right)^{2}},
$$

where $\epsilon_{0}$ is the vacuum permittivity, $A$ is the electrode area, $g_{\text {eff }}$ the effective gap, $w(t)$ the displacement of the plate, and $V(t)$ the applied voltage at time $t$. If both a DC bias voltage $V_{\mathrm{dc}}$ and a harmonic excitation voltage $V_{\mathrm{ac}}(t)=V_{\mathrm{ac}} \cos \left(\omega_{0} t\right)$ are applied, the voltage term in the electrostatic force becomes

$$
V^{2}(t)=V_{\mathrm{dc}}^{2}+\frac{1}{2} V_{\mathrm{ac}}^{2}+\frac{1}{2} V_{\mathrm{ac}}^{2} \cos \left(2 \omega_{0} t\right)+2 V_{\mathrm{dc}} V_{\mathrm{ac}} \cos \left(\omega_{0} t\right),
$$

with $\omega_{0}$ being the harmonic angular frequency. There are two time-dependent terms: $2 V_{\mathrm{dc}} V_{\mathrm{ac}} \cos \left(\omega_{0} t\right)$ and $\frac{1}{2} V_{\mathrm{ac}}^{2} \cos \left(2 \omega_{0} t\right)$. The first term is responsible for the pressure generated at the fundamental frequency $f_{0}=2 \pi \omega_{0}$ and is equally proportional to both $V_{\mathrm{dc}}$ and $V_{\mathrm{ac}}$. The second term generates pressure at the second harmonic frequency $2 f_{0}$ and is proportional to $V_{\text {ac }}$ squared. This inherent nonlinear behavior of the CMUT is naturally highly unwanted for harmonic imaging, as it is difficult to dissociate the harmonics generated in the tissue from the harmonic content of the transmitted signal. The plate displacement $w(t)$ in (1) is in addition nonlinear and is contributing to the intrinsic generation of harmonics by the CMUT itself. The level of generated second harmonic pressure has been investigated for varying DC and AC voltages, with a maximum achievable harmonic to fundamental amplitude ratio (HFR) of $-12 \mathrm{~dB}$, clearly emphasizing the CMUTs inherent limitations in harmonic imaging [2].

The nonlinearity caused by the voltage term can be eliminated by operating the CMUT without a bias voltage and with an excitation signal at half the fundamental frequency $\left(f_{0} / 2\right)$. Removing the bias voltage, however, significantly reduces the coupling coefficient. This can in principle be counterbalanced in transmit by significantly increasing the excitation voltage, but the low coupling will inevitably decrease the receive sensitivity considerably. In addition, removing the bias voltage does not eliminate the nonlinear contribution from the plate displacement $w(t)$. Other approaches such as the use of precompensated excitation waveforms [1], [3] and gap feedback linearization [4] for suppressing the intrinsic generated harmonic of the CMUT have been demonstrated. Most current ultrasound scanning equipment does, however, not facilitate these required advanced excitation signals. Alternative pulseshape has proven successful [5], but with a requirement of individual transducer calibration. Both requirements pose an issue in successful commercialization of a CMUT based probe for harmonic imaging. In this work, it is demonstrated how proper pulse coding of a bipolar pulser, which is present in most commercial ultrasound scanners, can significantly reduce the intrinsic second harmonic content of the pressure emitted by a generic CMUT. 

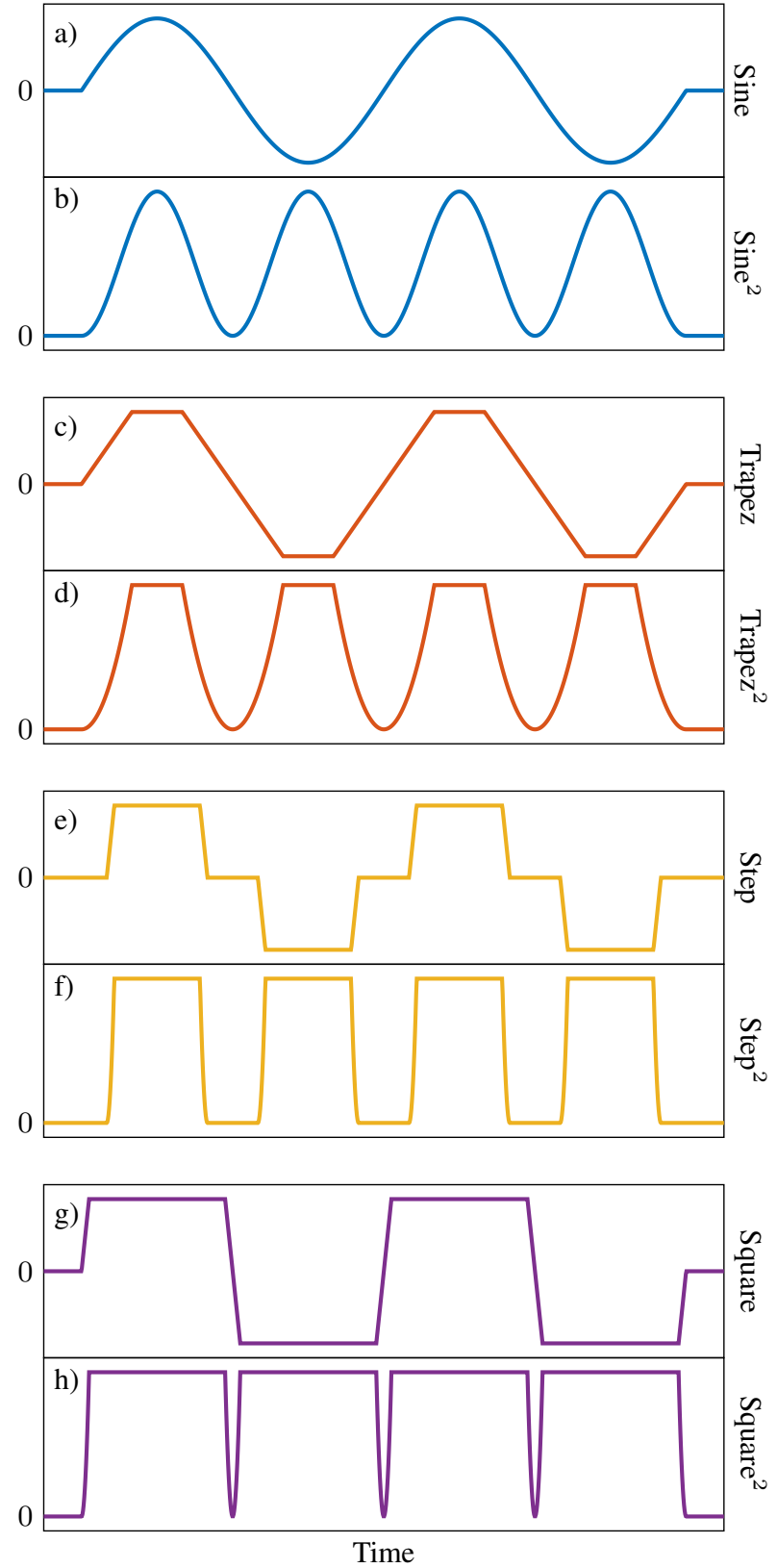

Fig. 1. Two period pulse-shapes and their squared outcomes: a) and b) sinusoidal, c) and d) trapezoidal, e) and f) step, g) and h) square. The sinusoidal, trapezoidal and step pulse-shapes all have higher frequency components when squared. The square function however contains almost no higher order frequency components.

\section{Pulse-Shapes}

A variety of different periodic pulse-shapes are used in ultrasound depending on the choice of pulser. Using a bipolar $5 \mathrm{MHz}$ sinusoidal pulse-shape as shown in Fig. 1a), gives the highest transferred power at the fundamental frequency. However, the non-linear squared voltage term in (2) means the CMUT will also experience a force at twice the fundamental frequency as depicted by the squared sinusoidal signal in Fig. 1b) and the Fourier spectrum of the squared pulse in

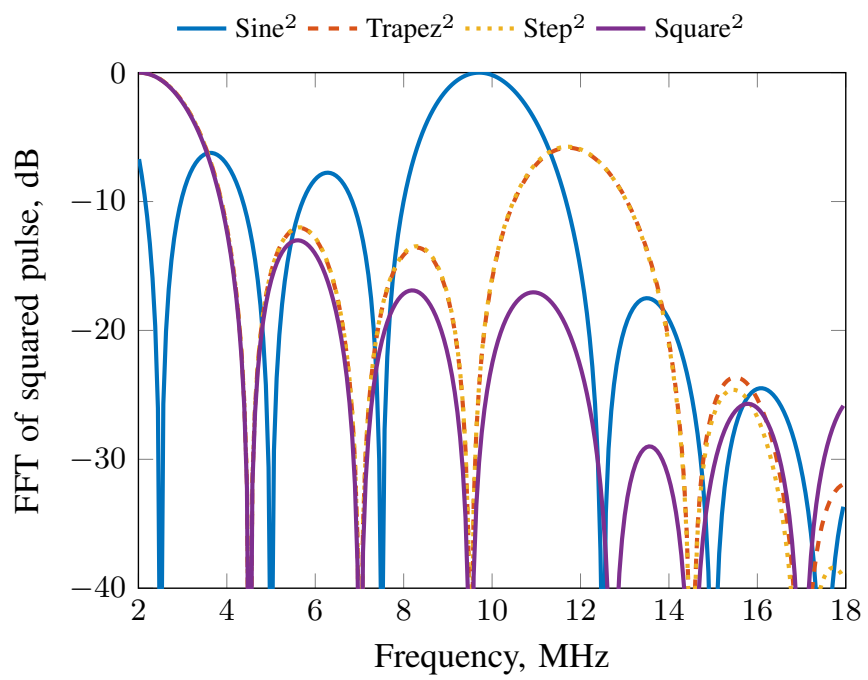

Fig. 2. Fourier spectrum of the squared $5 \mathrm{MHz}$ pulse-shapes showed in Fig. (b), d), f) and h) respectively. The squared sinusoidal pulse has a clear component at twice the fundamental frequency. The trapezoidal and step pulses likewise have a significant higher frequency component but at $12 \mathrm{MHz}$. The square pulse, however, shows a significantly reduction is higher order components, making it suitable for harmonic imaging.

Fig. 2. Another used pulse-shape is the bipolar trapezoidal plotted in Fig. 1c). The rise/fall time for trapezoidal pulse is $1 / 3$ of half the wavelength to reduce power loss at $3 \mathrm{rd}$ and 5th order harmonics. The squared outcome of the trapezoidal pulse (Fig. 1d)) also generates forces at higher frequency, more specifically around $12 \mathrm{MHz}$ as seen in the spectrum Fig. 2. A common pulse-shape for a commercial pulser is the step pulse seen in Fig. 1e) with a duty-cycle of $66.6 \%$ and a $5 \%$ of half the wavelength rise/fall time. The squared outcome of the step pulse (Fig. 1f) is similar to that of the trapezoidal pulse with a peak at $12 \mathrm{MHz}$ in the spectrum, Fig. 2. A bipolar tri-level pulser capable of generating the step pulse depicted in Fig. 1e) can also be used to generate a square pulse-shape seen in Fig. $1 \mathrm{~g}$ ) with a $5 \%$ of half the wavelength rise/fall time. The squared outcome of the square pulse seen in Fig. 1h) ends up being a low frequency square pulse (at the length of the pulse train) overlaid with narrow square pulses at $12 \mathrm{MHz}$. The very short duration (depending on the rise and fall slopes) of the $12 \mathrm{MHz}$ pulses reduces the energy of these pulses, and thus the amount of second harmonic. The result in the Fourier spectrum is clearly that higher order frequency components are highly reduced, when using a square pulse over the other pulse-shapes: sinusoidal, trapezoidal, and step.

\section{EXPERIMENTAL METHOD}

The effect of using a sinusoidal and square pulse excitation, respectively, are tested on a CMUT fabricated using wafer bonding and double local oxidation similar to the process presented in [6]. The linear CMUT array consists of 128 elements with an element width of $296 \mu \mathrm{m}$ and elevation height of $5 \mathrm{~mm}$. Each element contains 546 closely packed circular 
CMUT cells with a radius of $24.3 \mu \mathrm{m}$ resulting in a fill factor of $69 \%$. The CMUT plate thickness is $1.8 \mu \mathrm{m}$. The bump formed in the cavity of the CMUT by the double local oxidation has a radius comprising $2 / 3$ of the cell plate. The center frequency in water immersion is $5 \mathrm{MHz}$ and the pull-in voltage $V_{\mathrm{pi}}$ is $220 \mathrm{~V}$. The interconnect is identical to previous presented work [7] and the CMUT is coated with a $\sim 1 \mu \mathrm{m}$ layer of polydimethylsiloxane (PDMS) for electrical insulation. The PDMS layer is flat and has no lens effect.

The measurements with a sine pulse-shape were performed in a water tank using the experimental Synthetic Aperture Real-time Ultrasound System (SARUS) [8]. The hydrophone used was a HGL-0400 hydrophone connected to a AC-2010 pre-amplifier (Onda Corporation, CA, USA). The acoustic pressure of a plane wave emission from 12 elements was measured at $10 \mathrm{~mm}$ distance to the CMUT surface for a bias voltage of $160 \mathrm{~V}$ and varying $\mathrm{AC}$ voltage levels. The hydrophone was positioned at the center of the emitting CMUT element area. A two period $5 \mathrm{MHz}$ excitation was used, and the emission was repeated 10 times for each voltage level combination for noise reduction.

The measurements with a square pulse-shape were performed in a water tank using a commercial bipolar square pulser from Hitachi (HDL6V5582). The hydrophone used was a Optel $10 \mathrm{MHz}$ hydrophone (Optel Ultrasonic Technology, Poland). The acoustic pressure of a plane wave emission from 12 elements was measured at $10 \mathrm{~mm}$ distance to the CMUT surface for varying DC and AC voltage levels. The hydrophone was positioned at the center of the emitting CMUT element area. A two period $5 \mathrm{MHz}$ excitation was used, and the emission was repeated 10 times for each voltage level combination for noise reduction.

\section{A. Calculation of HFR level af CMUT surface}

The measured acoustic response of the hydrophone consist of both the fundamental $\left(f_{0}\right)$ and harmonic $\left(2 f_{0}\right)$ parts. To evaluate the fundamental pressure at the CMUT surface, the medium attenuation and loss of energy to higher harmonics must be added. The plane wave emission and absence of focusing lens allows for direct mapping without a diffraction compensation factor. Determining the second harmonic pressure at the CMUT surface likewise involves medium attenuation, but also the build-up of seconds harmonics caused by the nonlinear medium. For a pressure wave with constant energy (non-attenuating medium), the amplitudes of the harmonics are given by [9]

$$
p_{n}(\sigma)=\frac{2 p_{0}}{n \sigma} J_{n}(n \sigma),
$$

where $p_{0}$ is the unperturbed pressure, $n$ is the number of the harmonic, $J_{n}$ is the Bessel function of order $n$, and $\sigma=z / \bar{z}$ with

$$
\bar{z}=\frac{\rho_{0} c_{0}^{3}}{\beta \omega p_{0}},
$$

where $z$ is the propagation distance of the waveform, $\rho_{0}$ is the density of the undisturbed medium, $c_{0}$ is the speed of
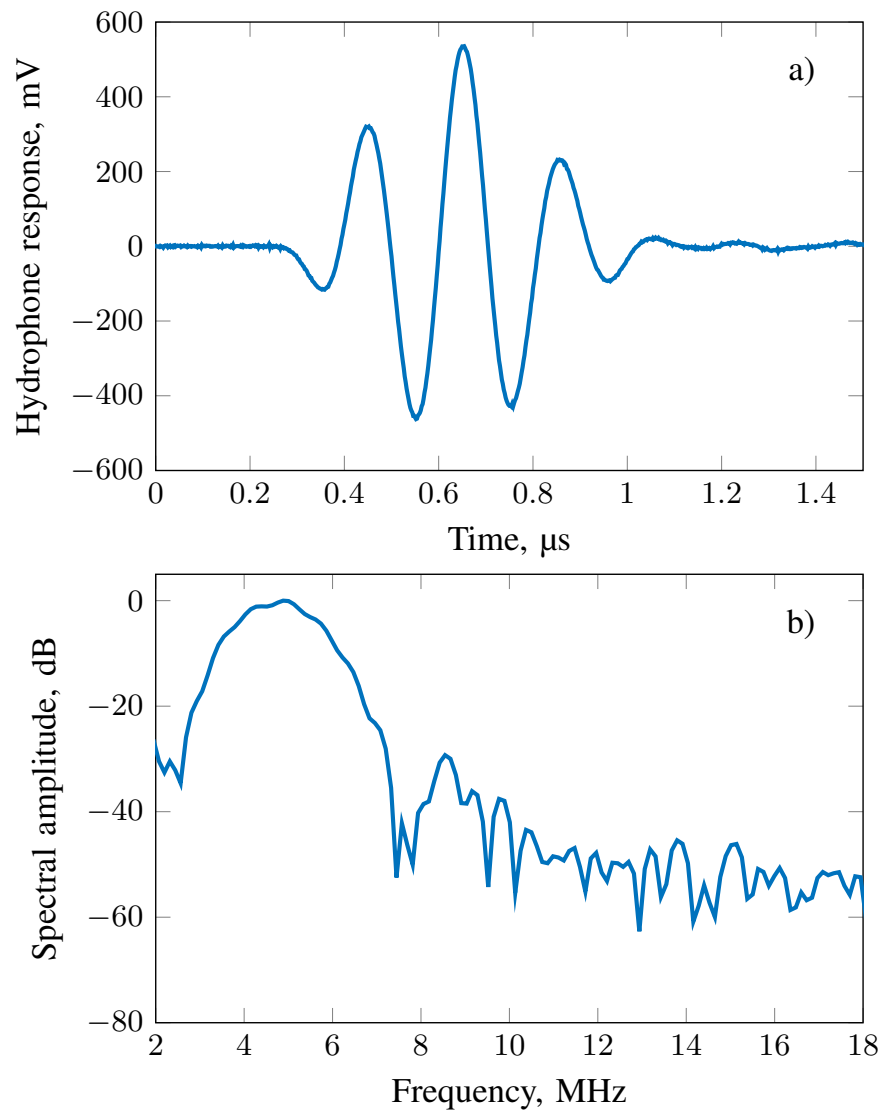

Fig. 3. a) hydrophone response and b) Fourier spectrum for a two period square pulse-shape with a bias voltage of $160 \mathrm{~V}$ and a peak excitation voltage of $65 \mathrm{~V}$. The spectrum shows the low second harmonic content of around $-40 \mathrm{~dB}$.

sound for linear waves, $\beta$ is the nonlinearity parameter (3.6 for water), and $\omega$ is the angular frequency of the transmitted plane wave. The fundamental and second harmonic amplitudes are determined separately from the measured acoustic signal by using a low-pass and high-pass digital filter respectively. Ignoring the medium attenuation of $0.0543 \mathrm{~dB} \mathrm{~cm}^{-1}$ at $5 \mathrm{MHz}$, the fundamental surface pressure can be calculated directly from (3) as the unperturbed pressure $p_{0}$. The second harmonic surface pressure $p_{\mathrm{HSP}}$ can subsequently be calculated from

$$
P_{2}=p_{2}+p_{\mathrm{HSP}}-\alpha,
$$

where $P_{2}$ is the second harmonic pressure measured at $10 \mathrm{~mm}$. $p_{2}$ is the second harmonic pressure generated in the medium (calculated from (3) using $p_{0}$ ) and $\alpha$ is the attenuation of $0.2170 \mathrm{~dB} \mathrm{~cm}^{-1}$ at $10 \mathrm{MHz}$. The harmonic to fundamental amplitude ratio at the CMUT surface can then be calculated by

$$
\mathrm{HFR}=p_{\mathrm{HSP}} / p_{0} .
$$

\section{EXPERIMENTAL RESULTS AND DISCUSSION}

The generated pressure is measured for both the two period sinusoidal and square pulse-shape with excitation voltages 


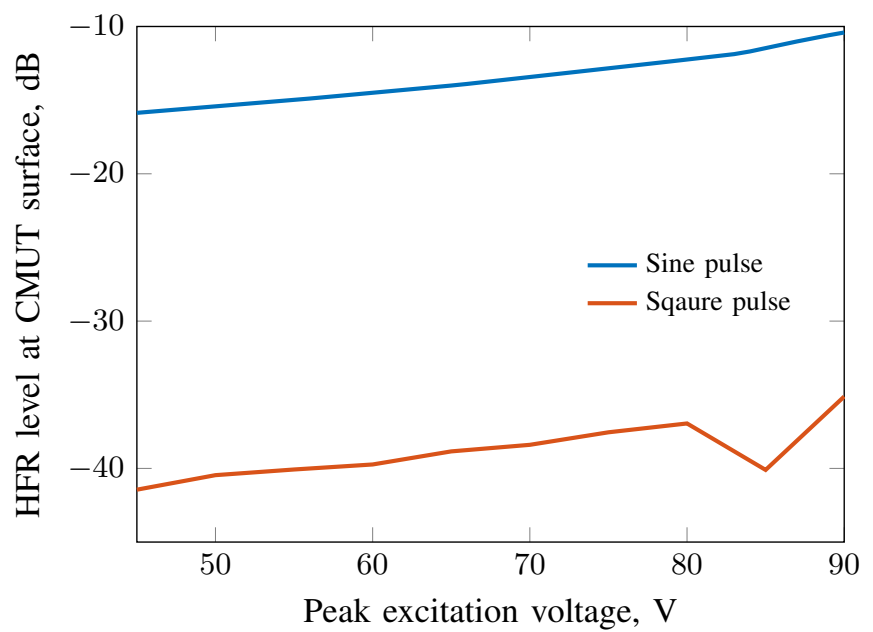

Fig. 4. Comparison of the harmonic to fundamental amplitude ratio for a two period sinusoidal and square pulse-shape, respectively. The peak excitation voltage is varied from $45 \mathrm{~V}$ to $90 \mathrm{~V}$ with a constant bias voltage of $160 \mathrm{~V}$. With a HFR level $25 \mathrm{~dB}$ lower than for the sinusoidal pulse, it is demonstrated how the square pulse efficiently can reduce the issues of intrinsic second harmonic generation in a CMUT.

varying from $45 \mathrm{~V}$ to $90 \mathrm{~V}$ and a constant bias voltage of $160 \mathrm{~V}$. The hydrophone response for the square pulse-shape with excitation voltage of $65 \mathrm{~V}$ is plotted in Fig. 3a). Fig. 3b) contains the corresponding spectrum of the signal response, and shows the main frequency peak at $5 \mathrm{MHz}$ and the low level of the higher harmonics. The calculated harmonic to fundamental amplitude ratio is for both pulse-shapes plotted in Fig. 4 as function of the varied excitation voltage. While the HFR level for the sinusoidal pulse is between $-15 \mathrm{~dB}$ and $-10 \mathrm{~dB}$ the level is decreased around $25 \mathrm{~dB}$ to between $-40 \mathrm{~dB}$ and $-35 \mathrm{~dB}$ for the square pulse-shape. Not only is bipolar square pulser readily available commercially, it is also a versatile and easily implementable method for reducing the issues of intrinsic harmonic generation in CMUTs.

\section{CONCLUSION}

The squared relation between the generated pressure and applied excitation voltage of the CMUT, means that periodic pulse-shapes such as sinusoidal, trapezoidal and step functions all gives higher order frequency components resulting in high harmonic to fundamental amplitude ratios. It is shown, both analytically and experimentally, how a proper coded square pulse, from a commercially available bipolar pulser, can significantly reduce the intrinsic harmonic content of the CMUT. The difference in HFR level between a sinusoidal and sqaure pulse-shape is examined with hydrophone measurements at $10 \mathrm{~mm}$ distance in a water tank with $5 \mathrm{MHz}$ plane wave emission and compensated to evaluate surface pressures. The HFR level for the sinusoidal pulse is between $-15 \mathrm{~dB}$ and $-10 \mathrm{~dB}$ depending on the excitation voltage magnitude. For the square pulse-shape the HFR level is measured to between $-40 \mathrm{~dB}$ and $-35 \mathrm{~dB}$, a level witch can enable CMUT transducer use in clinical applications. A reduction achieved without the need for complex multi stage linear transmitters for advanced waveforms or specific transducer tuned pulse-shapes.

\section{ACKNOWLEDGEMENT}

This work is financially supported by the Danish National Advanced Technology Foundation (82-2012-4) and by BK Ultrasound.

\section{REFERENCES}

[1] A. Novell, M. Legros, N. Felix, and A. Bouakaz, "Exploitation of capacitive micromachined transducers for nonlinear ultrasound imaging," IEEE Transactions on Ultrasonics, Ferroelectrics, and Frequency Control, vol. 56, no. 12, pp. 2733-2743, Dec. 2009.

[2] A. Lei, S. E. Diederichsen, S. M. Hansen, M. B. Stuart, H. Bouzari, J. A. Jensen, and E. V. Thomsen, "Output pressure and harmonic characteristics of a CMUT as function of bias and excitation voltage," in Ultrasonics Symposium (IUS), 2015 IEEE International, Oct. 2015, pp. 1-4.

[3] M. Legros, A. Novell, A. Bouakaz, G. Ferin, R. Dufait, and D. Certon, "Tissue harmonic imaging with CMUTs," in Ultrasonics Symposium (IUS), 2011 IEEE International, Oct. 2011, pp. 2249-2252.

[4] S. Satir and F. L. Degertekin, "Harmonic Reduction in Capacitive Micromachined Ultrasonic Transducers by Gap Feedback Linearization," Ieee Transactions on Ultrasonics, Ferroelectrics, and Frequency Control, vol. 59, no. 1, pp. 50-59, Jan. 2012.

[5] A. S. Savoia, G. Scaglione, G. Caliano, A. Mazzanti, M. Sautto, and F. Quaglia, "Second-harmonic reduction in CMUTs using unipolar pulsers," in Ultrasonics Symposium (IUS), 2015 IEEE International, Oct. 2015, pp. 1-4.

[6] K. K. Park, H. Lee, M. Kupnik, and B. T. Khuri-Yakub, "Fabrication of Capacitive Micromachined Ultrasonic Transducers via Local Oxidation and Direct Wafer Bonding," Journal of Microelectromechanical Systems, vol. 20 , no. 1, pp. 95-103, Feb. 2011.

[7] M. F. la Cour, M. B. Stuart, M. B. Laursen, S. E. Diederichsen, E. V. Thomsen, and J. A. Jensen, "Investigation of PDMS as coating on CMUTs for imaging," in Ultrasonics Symposium (IUS), 2014 IEEE International, Sep. 2014, pp. 2584-2587.

[8] J. A. Jensen, H. Holten-Lund, R. T. Nilsson, M. Hansen, U. D. Larsen, R. P. Domsten, B. G. Tomov, M. B. Stuart, S. I. Nikolov, M. J. Pihl, Y. Du, J. H. Rasmussen, and M. F. Rasmussen, "SARUS: A synthetic aperture real-time ultrasound system," IEEE Transactions on Ultrasonics, Ferroelectrics, and Frequency Control, vol. 60, no. 9, pp. 1838-1852, Sep. 2013.

[9] A. D. Pierce, Acoustics: an introduction to its physical principles and applications, 1989th ed. Woodbury, N.Y: Acoustical Society of America, 1989. 